\title{
NUESTRA EXPERIENCIA EN LA ENFERMEDAD DE PEYRONIE CON LA TÉCNICA DE NESBIT.
}

José Luis Moyano Calvo, Javier Sánchez de la Vega, Javier Giraldez Puig, Guillermo Dávalos Casanova, Ignacio Huesa Martínez, José Luis Maestro Durán, Antonio Ramírez Mendoza y Antonio Morales López.

Centro Urológico San Ignacio. Sevilla. España.

\begin{abstract}
Resumen.- OBJETIVO: La técnica de Nesbit esa la técnica de referencia en la cirugía correctora de la incurvación penenana en la enfermedad de Peyronie. Presentamos nuestra experiencia en el Centro Urológico san Ignacio.
\end{abstract}

MÉTODO: 68 pacientes con incurvación peneana estable y que deseaban corrección quirúrgica de la misma. Se considera corrección de la curvatura cuando la rectificación es igual o superior al $80 \%$ de la angulación patológica. La edad media de los pacientes es de 44 años (31-77). Un 53\% presentaban dificultad para la penetración, un 20\% molestias en su pareja, un 27\% se intervinieron por razones estéticas. La desviación peneana era dorsal en el $42 \%$, izquierda en el $46 \%$ y derecha en el 12\%. El ángulo de incurvación medido en grados era menor de $30^{\circ}$ en el $28 \%$, entre 30 y 45 en el $36 \%$, entre 45 y 60 en el $37 \%$ y mayor de $60 \%$ en el $7 \%$.
RESULTADOS: El seguimiento medio de la serie es de 46 meses. Acortamiento peneano superior a $1,5 \mathrm{~cm}$ en el 20\% de los enfermos. El $85 \%$ de los enfermos están satisfechos con los resultados obtenidos. La queja más frecuente es el acortamiento peneano. Se ha corregido la desviación en el 92\% de los pacientes. Hemos reintervenido a cuatro pacientes, A dos por fimosis marcada a los tres meses de la intervención, los otros dos enfermos presentaron recidiva de la curvatura.

CONCLUSIONES: La técnica de Nesbit es una técnica sencilla, con escasas complicaciones y buenos resultados tanto en corrección de curvatura como en satisfacción del enfermo.

Palabras clave: Nesbit. Peyronie. Pene. Curvatura.

Summary.- OBJECTIVES: The Nesbit technique is the reference technique for the surgical correction of the penile incurvation of Peyronie's disease. We report our experience at the Centro Urológico San Ignacio.

METHODS: 68 patients with stable penile incurvation who requested surgical correction. Correction of the curvature is defined as $\geq 80 \%$ rectification of the pathologic angle. Mean patient age was 44 years (3 1-77). 53\% of the patients presented difficulties for penetration, 20\% partner's discomfort on intercourse, and $27 \%$ requested surgery for aesthetic reasons. Penile deviation was dorsal in $42 \%$ of the patients, to the left in $46 \%$ and to the right in $12 \%$. The curvature angle was less than $30 \%$ in $28 \%$, between $30^{\circ}-45^{\circ}$ in $36 \%$, between $45^{\circ}-60^{\circ}$ in $37 \%$ and $>60^{\circ}$ in $7 \%$.

RESULTS: Mean follow-up of the series was 36 months. $20 \%$ of the patients had a penile shortening longer than $1.5 \mathrm{~cm} .85 \%$ of the patients are satisfied with the results.
José Luis Moyano Calvo Centro Urológico San Ignacio C/Eduardo Rivas no 3 Sevilla 41018 (España) joseluismoyano@ya.com Trabajo recibido: 21 de noviembre 2005 
The most frequent complaint was penile shortening. Correction was achieved in $92 \%$ of the patients. Four patients were reoperated: two of them for significant phimosis three months after surgery, and the other two for curvature recurrence.

CONCLUSIONS: The Nesbit technique is a simple technique, with a low complication rate, and good results both in curvature correction and patient satisfaction.

Keywords: Nesbit. Peyronie. Penis. Incurvation.

\section{INTRODUCCIÓN}

En 1965 Nesbit describió una técnica quirúrgica para corregir la incurvadura congénita peneana (1). Esta técnica fue aplicada por Prior en 1977 para corregir la curvatura en la enfermedad de Peyronie (2). Desde entonces esta técnica ha sido la referencia con la que se han comparado el resto de las técnicas.

Se han publicado numerosas modificaciones técnicas, pero el principio básico, corregir por el lado convexo, se mantiene inmutable, así como la mayoría de los pasos quirúrgicos.

Presentamos nuestra experiencia en el Centro Urológico San Ignacio de Sevilla.

\section{MATERIAL Y MÉTODO}

Desde 1998 hemos sido intervenidos 68 pacientes que presentaban incurvación peneana debido a la enfermedad de Peyronie.

Los criterios para realizar la intervención fueron:

- Curvadura estable más de seis meses

- Capacidad eréctil conservada

- Dificultad o incomodidad para la practica del coito o razones estéticas

A todos los enfermos se les solicitó fotos del pene en erección para medir ángulos según Kelami (3)

Se considera corrección de la curvatura cuando la rectificación es igual o superior al $80 \%$ de la angulación patológica.

La técnica quirúrgica es similar a la descrita por Nesbit (1). Los limites de la elipse que vamos a resecar se determinan marcando los extremos laterales del pliegue formado por la pinza de Allis y las indentaciones que la misma deja en la albugínea al retirarla. Suturamos el defecto con puntos sueltos y nudo invertido de ácido poliglicólico 3/0.

El enfermo es dado de alta al día siguiente y se le aconseja evitar relaciones sexuales y erecciones en un mes, con progresiva reanudación de su actividad sexual.

La edad media de los pacientes es de 44 años (31-77).

Las razones por las que los enfermos decidieron intervenirse fueron:

- Un 53\% presentaban dificultad para la penetración - Un $20 \%$ molestias en su pareja

- Un $27 \%$ se intervinieron por razones estéticas

La desviación peneana la valoramos según el mayor predominio de la curvatura:

$\begin{array}{ll}\text { - Dorsal } & 42 \% \\ \text { - Izquierda } & 46 \% \\ \text { - Derecha } & 12 \%\end{array}$

El ángulo de incurvación medido en grados:

$\begin{array}{lr}\text { - }<30^{\circ} & 28 \% \\ \text { - } 30^{\circ}-45^{\circ} & 36 \% \\ \text { - } 45^{\circ}-60^{\circ} & 37 \% \\ \text { - }>60^{\circ} & 7 \%\end{array}$

\section{RESULTADOS}

El seguimiento medio de la serie es de 46 meses. El número medio de elipses resecadas por enfermo es de dos, oscilando entre uno y cuatro.

Acortamiento peneano superior a $1,5 \mathrm{~cm}$ en el $20 \%$ de los enfermos.

El $85 \%$ de los enfermos están satisfechos con los resultados obtenidos. La queja más frecuente entre los que no están satisfechos es el acortamiento peneano. los pacientes.

Se ha corregido la desviación en el 92\% de Hemos reintervenido a cuatro pacientes:

- A dos se les reintervino por fimosis marcada a los tres meses de la intervención. Estos enfermos habían solicitado no ser circuncidados. 
- Dos enfermos presentaron recidiva de la curvatura, uno de ellos antes del mes (coito a los quince días de la intervención) y el otro a los nueve meses. Tras la reintervención ambos enfermos estás satisfechos y con la curvatura corregida

\section{DISCUSIÓN}

En el tratamiento quirúrgico de la enfermedad de Peyronie, el objetivo fundamental es conseguir un pene funcional que permita la práctica del coito sin causar molestias ni en el enfermo ni en su pareja. La técnica quirúrgica descrita por Nesbit consigue este objetivo sin actuar sobre la placa, consiguiendo la corrección de la curvatura resecando un fragmento de albugínea en el lado convexo.

Esta resección conlleva un acortamiento peneano que es la principal causa de queja de los enfermos. Sin embargo, como señala Andrews (4) dicho acortamiento raramente interfiere en la vida sexual, además la mayoría de los pacientes recuerdan el tamaño peneano de cuando eran jóvenes.

La técnica de Nesbit es una técnica segura, con una tasa alta de satisfacción y de corrección de la curvatura, superior en la mayoría de las series al $80 \%$. Además ha superado la prueba del tiempo, con seguimientos superiores a los cinco años, llegando en algunos casos a más de diez años (5). (Tabla I).
Para conseguir estos resultados es muy importante la indicación de la cirugía. Para ello hay que observar varios factores:

\section{Tipo de deformidad y angulación}

La intervención de Nesbit está indicada en penes con buena capacidad eréctil, adecuada longitud peneana y ángulo menor de $60 \%$ sin alteraciones en la forma del pene.

Si el pene tiene deformación en reloj de arena o se pliega alrededor de un eje o con ángulo mayor de 60\%, es mejor en estos casos realizar excisión de la placa y colocación de un parche o bien incisión de la placa con colocación de parche (14-16)

\section{Estabilidad de la curvatura}

No existe ninguna forma de saber si la enfermedad esta en la fase estable y por tanto la curvatura peneana no aumentará su ángulo. Normalmente la enfermedad se estabiliza en doce-dieciséis meses tras su aparición. La mayoría de los autores están de acuerdo en esperar al mernos cuatro meses desde que la curvatura está estable.

Andrews (4) revisa 51 pacientes en los que la técnica de Nesbit ha fallado, y en el $39 \%$ de los mismos la curvatura ha recidivado nuevamente más de $30^{\circ}$ tras once meses de cirugía y tras haber descartado fallo en la técnica quirúrgica. Por ello insiste en que es muy importante garantizar que la enfermedad esta en la fase estable.

\section{TABLA I. RESULTADOS DE LA TÉCNICA DE NESBIT (\%).}

\begin{tabular}{|l|l|l|l|l|l|l|l|}
\hline Autor & Año & N & Corrección & Satisfecho & D. Eréctil & Acortamiento & Seguimiento \\
\hline Poulsen & 1995 & 57 & 88 & & & & $6-60$ \\
\hline Litch & 1997 & 28 & 79 & 79 & 4 & 37 & 12 \\
\hline Pryor & 1998 & 359 & 82 & & & 5 & $>120$ \\
\hline Brake & 1999 & 20 & 71 & 50 & & & 45 \\
\hline Daitch & 1999 & 19 & 93 & 79 & 7 & 57 & 24 \\
\hline Syed & 2003 & 42 & 90 & 76 & 12 & 37 & 84 \\
\hline R. Tolrá & 2003 & 45 & 88,8 & 86,6 & 0 & & 63 \\
\hline Savoca & 2004 & 279 & 86 & 84 & 12,9 & 17 & 89 \\
\hline Rolle & 2005 & 18 & 100 & 80 & 0 & 77 & $>12$ \\
\hline C.U.S.I. & 2005 & 68 & 92 & 85 & 0 & & 36 \\
\hline
\end{tabular}


Por esta razón nosotros no intervenimos a pacientes en las que de manera objetiva la curvatura no ha variado en al menos seis meses. A pesar de ello hemos tenido que reintervenir a dos pacientes por recidiva en la curvatura de los cuales solo uno es achacable a recidiva de la intervención.

La tasa de reoperaciones por recidiva de la curvatura es baja, en nuestra experiencia un 3\%, Poulsen (6) describe un 5\%. La serie de Andrews se basa en una mayor de 359 enfermos (17) por lo que su tasa de reintervenciones sería de 8,6\%.

\section{Valorar la potencia sexual previa a la cirugía}

Creemos, de acuerdo con la mayoría de los autores $(4,10,12,13)$ que la realización de pruebas para objetivar la capacidad eréctil (registro de erecciones nocturnas, eco-doppler con inyección de PGE 1) debe realizarse en caso de duda y no de manera rutinaria, aunque hay algunos autores que sí las realizan $(9,16)$.

No hemos tenido hasta ahora ningún caso de disfunción eréctil tras la intervención. Creemos que se debe a la selección de los enfermos, no realizando este tipo de cirugía en caso de disfunción eréctil que no responda a medicación oral.

Andrews (4) observa que el $60 \%$ de los pacientes que presentan disfunción eréctil tras cirugía ya presentaban dificultad en la erección previa a la intervención que no respondía a terapia habitual.

Rolle (13) con criterios muy similares a los nuestros tampoco observa disfunción eréctil en su serie, si bien su seguimiento es corto. Savoca (12) con un seguimiento más largo observa la aparición de disfunción eréctil a lo largo del tiempo, sin poder establecer relación con la cirugía y sí con otras causas conocidas de disfunción (diabetes, causas vasculares).

Dentro de las complicaciones descritas (hematomas, pérdida de sensibilidad en glande, herniaciones, granulomas, lesiones uretrales, fimosis) sólo hemos tenido dos enfermos ( $3 \%$ de nuestra serie) con fimosis tras cirugía. Savoca (12) tiene que reintervenir por esta causa al $10 \%$ de los enfermos a los que no les realiza circuncisión y Syed (10) al 3,5\% de su serie. Para evitar esta pequeña pero enojosa complicación la circuncisión es practicada en todos los enfermos, sin dar opción a conservar el prepucio, ya que en nuestra experiencia el $100 \%$ de los enfermos a los que se les ha conservado el prepucio han tenido que ser reintervenidos.

La queja fundamental de los enfermos es el acortamiento peneano. Se calcula que se acorta 1.
$2 \mathrm{~mm}$ por cada $10^{\circ}$ de incurvación corregida. Andrews (4) aprecia acortamiento superior a dos $\mathrm{cm}$ en diecinueve enfermos, el $6 \%$ de su serie; Savoca (12) un $17 \%$ (entre 1,5 y $3 \mathrm{~cm}$ ) y Rolle (13) en un $77 \%$. En nuestra experiencia aunque un $15 \%$ se queja de acortamiento peneano en ninguno de ellos interfiere con su actividad sexual.

La comparación de la técnica de Nesbit con la plicatura ha sido analizada por varios autores, Poulsen (6) la considera superior, con mejores resultados en su conjunto $93 \%$ frente a $63 \%$ siendo la complicación más frecuente la falta de erección postoperatoria. Brake (8) también encuentra mejores resultados $(71 \%$ frente a $36 \%)$ y menor tasa de recidivas (18\% frente a $56 \%$ ) con la técnica de Nesbit., Scultheiss (18) encuentra mayor tasa de recidiva con la técnica de plicatura.

\section{CONCLUSIÓN}

La técnica de Nesbit es una técnica sencilla, con escasas complicaciones y buenos resultados tanto en corrección de curvatura como en satisfacción del enfermo.

\section{BIBLIOGRAFIA y LECTURAS RECOMENDADAS ( ${ }^{*}$ lectura de interés $y^{* *}$ lectura fundamental)}

1. NESBIT, R.H.: "Congenital curvature of the phallus: Report of three cases with description of corrective operation". J. Urol., 93: 230, 1965.

2. PRYOR, J.P.; FITZPATRICK, J.M.: "A new approach to the correction of the penile deformity in Peyronie's disease”. J. Urol., 122: 622, 1979.

*3. KELAMI, A.: "Autophotography in evaluation of functional penile disorders". Urology, 21: 628, 1983.

*4. ANDREWS, H.O.; AL-AKRAA, M.; PRYOR, J.P. y cols.: "The nesbit operation for Peyronie's disease: an analysis of the failures". BJU Int., 87: 658, 2001.

5. PRYOR, J.P.: "Correction of penile curvature and Peyronie's disease: why I prefer the Nesbit technique". Int. J. Impot. Res., 10: 129, 1998.

6. POULSEN, J.P.; KIERKEBY, H.J.: "Treatment of penile curvature--a retrospective study of 175 patients operated with plication of the tunica albuginea or with the Nesbit procedure". Br. J. Urol., 75: 370, 1995. 
7. LITCH, R.M.; LEWIS, R.W.: "Modified Nesbit porcedure for the tratment of Peyronie's disease: acomparative outcome analysis". J. Urol., 158: 460, 1997.

8. BRAKE, M.; KELLER, H.; LAMADE, F. y cols.: "Surgical correction of penile deviation. Nesbit vs. Schroeder-Essed method". Urologe A. 38: 264,L 1999.

9. DAITCH, J.A.; ANGERMEIER, K.W.; MONTAGUE, D.K.: "Modified corporoplasty for penile curvature; long-term results and patient satisfaction". J. Urol., 162: 2006, 1999.

*10. SYED, A.H.; ABBASI, Z.; HARGREAVE, T.B.: "Nesbit procedure for disabling Peyronie's curvature: a median follow-up of 84 months". Urology, 61: 999, 2003.

11. RODRÍGUEZ TOLRÁ, J.; FRANCO MIRANDA, E.; PRATS PUIG, J.M. y cols.: "Tratamiento con la técnica de Nesbit en pacientes con la enfermedad de Peyronie". Actas Urol. Esp., 27: 803, 2003.

*12. SAVOCA, G.; SCIERI, F.; PIETROPAOLO, F. y cols.: "Straightening corporoplasty for Peyronie's disease: a review of 218 patients with median follow-up of 89 months". Eur. Urol., 46: 610, 2004.

13. ROLLE, L.; TAMAGNONE, A.; TIMPANO, M. y cols.: "The Nesbit operation for penile curvation: an esay and effective technical modification". J. Urol., 173: 171, 2005.

14. LEVINE, L.A.; LENTING, E.L.: "A surgical algorithm for the tratment of peyronie's disease". J. Urol., 158: 2149, 1997.

15. MONTORSI, F.; SALONIA, A.; MAGA, T. y cols.: "Evidence based assesment of long-term results of plaque incision and vein grafting for Peyroniés disease". J. Urol., 163: 1704, 2000.

16. GHOLAMI, S.; GONZALEZ-CADAVID, N.F.; LIN, C.S. y cols.: "Peyronie's disease: a review". J. Urol., 169: 1234, 2003.

17. RALPH, D.J.; AL-AKRAA, M.; PRYOR, J.P.: "The Nesbit operation for Peyronie's disease: 16 years experience". J. Urol., 154: 1362, 1995.

18. SCHULTHEISS, D.; MESCHI, M.R.; HAGEMANN, J.: "Congenital and acquired penile deviation treated with the essed plication method". Eur. Urol., 38: 167, 2000. 